\title{
The Complex Upper HR Diagram
}

\section{Roberta M. Humphreys}

Minnesota Institute for Astrophysics, School of Physics and Astronomy, 116 Church St. SE, University of Minnesota, Minneapolis, MN 55455, USA; roberta@umn.edu

Received: 24 July 2019; Accepted: 14 August 2019; Published: 23 August 2019

\begin{abstract}
Several decades of observations of the most massive and most luminous stars have revealed a complex upper HR Diagram, shaped by mass loss, and inhabited by a variety of evolved stars exhibiting the consequences of their mass loss histories. This introductory review presents a brief historical overview of the HR Diagram for massive stars, highlighting some of the primary discoveries and results from their observation in nearby galaxies. The sections in this volume include reviews of our current understanding of different groups of evolved massive stars, all losing mass and in different stages of their evolution: the Luminous Blue Variables (LBVs), B[e] supergiants, the warm hypergiants, Wolf-Rayet stars, and the population of $\mathrm{OB}$ stars and supergiants in the Magellanic Clouds.
\end{abstract}

Keywords: massive stars; local group; supergiants; Magellanic Clouds; M31; M33

\section{Introduction}

The reviews and papers in this Special Issue focus on the properties of the most luminous stars in nearby galaxies, those galaxies in which the brightest individual stars are resolved and can be observed. The most luminous stars are also the most massive and because of their intrinsic brightness and relatively short lifetimes they provide our first probes of the progress of stellar evolution in different environments.

The study of massive stars in other galaxies offers many advantages. Foremost of course is distance. Studies of stellar populations in nearby galaxies have the advantage that all the stars are at approximately the same distance, a distance that is relatively well known, especially in comparison with the uncertain distances of individual stars in our own galaxy. In the Milky Way, our observations are also limited to a relatively small volume by interstellar extinction, which can be high and uncertain at increasing distances. In external galaxies, extinction by dust is still a problem but the foreground extinction is well determined from maps of the interstellar "cirrus" or dust along the line of sight. Internal extinction within the galaxy can be variable and must still be corrected.

In this introductory review, I present a brief historical overview with emphasis on some of the main developments in the study of massive stars in nearby galaxies. Some of the first work on stars in other galaxies was driven by the identification of Cepheids for the extragalactic distance scale, see, for example, the comprehensive survey of NGC 2403 by Tammann \& Sandage [1]. Other types of variables were also recognized including the first discussion of a class of luminous variables by Hubble \& Sandage [2] in the Local Group spirals M31 and M33 that we now call Luminous Blue Variables (LBVs). This work was done on photographic plates and the magnitudes of individual stars were often measured by hand.

The study of individual stars and their placement on the HR Diagram requires several types of data: accurate multicolor photometry for spectral energy distributions (SEDs) and the measurement of interstellar extinction; infrared observations of circumstellar dust and mass loss; and, most important, spectroscopy, for classification, temperatures, luminosity indicators, emission lines, and evidence for 
mass loss from line profiles. When observing stars in other galaxies, we also have to be concerned about foreground contamination: stars in the Milky Way seen projected against the distant galaxy. Photometry is not sufficient to remove these Galactic stars, especially those of intermediate temperature and late spectral type; spectra are required.

The primary galaxies discussed in this article are the Local Group spirals M31 and M33, and the Large and Small Magellanic Clouds due to their relative proximity and the number of surveys of their luminous stellar populations. Other Local Group galaxies are also included as well as results for stars in a few nearby spiral galaxies.

\section{Early Work-The Magellanic Clouds}

The early objective prism surveys by the Harvard College Observatory and the resulting Henry Draper Catalog and Extension (HD and HDE) provided the first survey with spectral types of the brightest stars in the Magellanic Clouds [3,4]. The HDE however did not cover the Small Cloud. Feast, Thackeray \& Wesselink [5] published the first detailed list of the brightest stars in the Magellanic Clouds with spectral classifications based on slit spectra of individual stars. Their paper included magnitudes, colors, positions, radial velocities and notes on the individual stars relative to emission lines and other features for 50 stars in the SMC and 105 in the LMC. The HDE catalog was the primary source for the LMC stars, plus stars selected as blue based on their colors in the 30 Dor region. The SMC list relied on "B" type stars from the HD plus emission line stars from the survey by Henize [6]. Their work did not include the red or M-type supergiants. The reddest stars in their study were the very luminous F- and G-type supergiants. Their HR Diagram, reproduced here in Figure 1, while not complete, shows the visually brightest stars with $\mathrm{M}_{v}$ approaching $-10 \mathrm{mag}$.

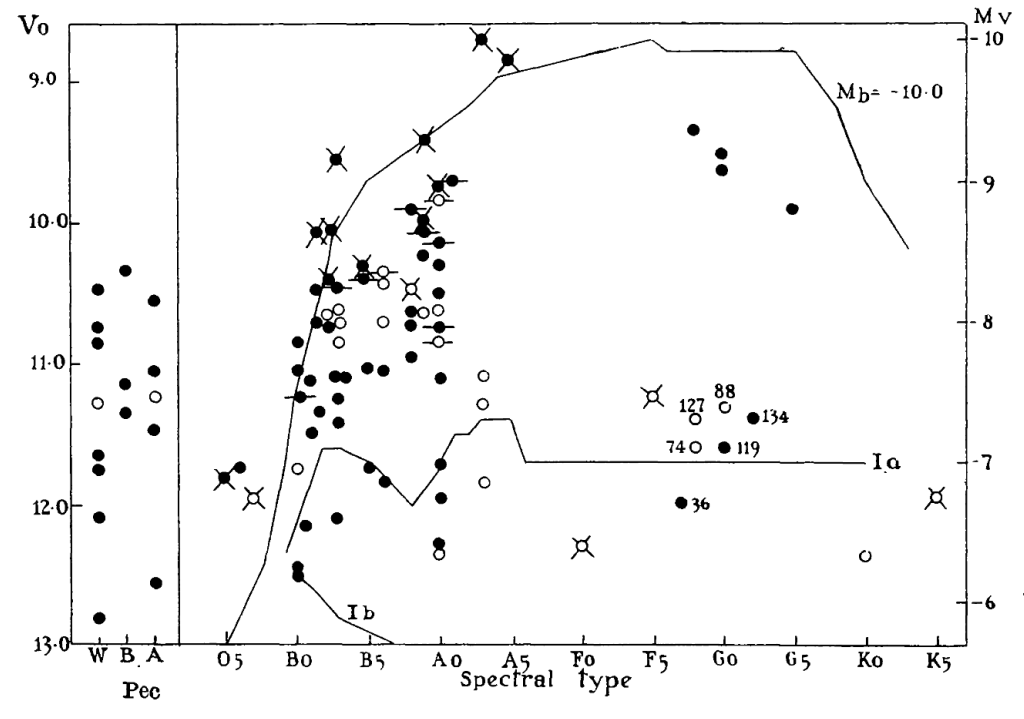

$L M C$.

FrG. 13.-HR Diagram of Cloud stars.

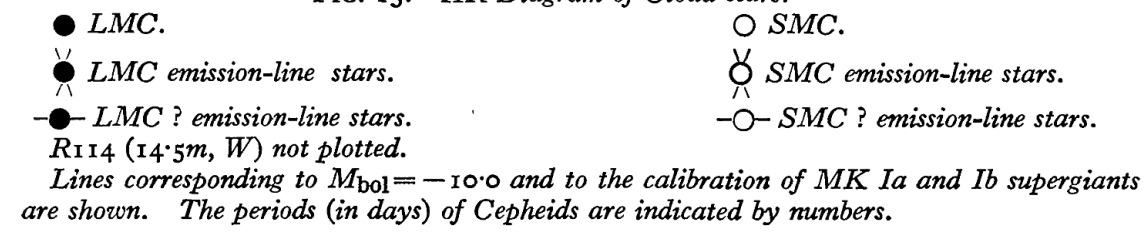

Figure 1. The HR Diagram for the brightest stars in the LMC and SMC from [5]. LMC stars are closed symbols and those in the SMC are shown as open symbols. 
Additional work on the fainter stellar populations of hot or OB-type stars and red M-type stars was provided by the lower resolution objective prism surveys throughout the 1960s and 1970s. These included surveys for OB type stars in the LMC and SMC by Sanduleak [7,8] and Fehrenbach \& Duflot [9] and for the M-type stars by Westerlund [10,11] and Blanco et al. [12,13]. These necessary photographic surveys provided extensive finding lists for further spectral classification leading to the first comprehensive HR Diagrams for these two galaxies and the comparison of their massive and luminous stellar populations with the Milky Way.

\section{The HR Diagram}

Fundamental data for the most luminous stars have application to numerous astrophysical questions specifically with respect to stellar evolution, the final stages of the most massive stars as the progenitors of supernovae, and the dependence of their basic parameters on the host galaxy. In the 1970s, there was also considerable interest in the luminosity calibration of the brightest stars and their potential as extragalactic distance indicators [14].

The massive star population in the Milky Way, although restricted to a relatively small volume, within $\approx 3 \mathrm{kpc}$ of the Sun, is critical as a reference population and as representative of our local region of the Galaxy, despite uncertain distances and possible incompleteness. Humphreys [15] published an HR Diagram for the Galactic supergiants and $\mathrm{O}$ stars with spectral types and photometry in stellar associations and clusters with known distances to derive their luminosities. For a more complete population in the upper HR Diagram, the less luminous early B-type main sequence and giant stars were also included. Compared to previous work, numerous surveys, especially of the Southern sky, had added greatly to the number of confirmed supergiants and $O$ stars [16-18] and red supergiants $[19,20]$. For comparison with evolutionary tracks, the derived absolute visual magnitudes and spectral types were transformed to absolute bolometric luminosities and effective temperatures based on the available calibrations.

Humphreys [21] subsequently published HR Diagrams for the LMC. The basic data for the confirmed supergiants and early-type stars came from the extensive catalogs of spectral types and photometry by Ardeberg et al. [22] and Brunet et al. [23], from Feast, Thackeray \& Wesselink [5], and from Walborn [24] for the early O-type stars. The data for the M-type supergiants came from the spectroscopic survey in the same paper.

An empirical comparison of these HR Diagrams, for the massive stars in our region of the Milky Way and for the LMC, revealed comparable populations of massive stars based on the distribution of their spectral types and luminosities across the HR Diagrams. The most important result was the recognition by Humphreys \& Davidson [25] of an empirical upper luminosity boundary or upper limit in the luminosity vs. temperature diagrams.

\section{The Humphreys-Davidson Limit}

The original HR Diagrams from the Humphreys and Davidson 1979 paper are reproduced here in Figures 2 and 3, with the upper boundary shown as a solid line. The original eyeball fit was first drawn to approximate the upper boundary of the supergiant luminosities in the Milky Way. The same line was then transferred to the LMC Diagram, which also matched the observed upper envelope to the LMC luminosities. The upper boundary for both galaxies is an envelope of declining luminosity and decreasing temperature for the hottest stars and a relatively tight upper limit to the luminosities of the cooler stars (less than $\approx 10,000 \mathrm{~K}$ ) near $\mathrm{M}_{B o l} \approx-9.5 \mathrm{Mag}$. 


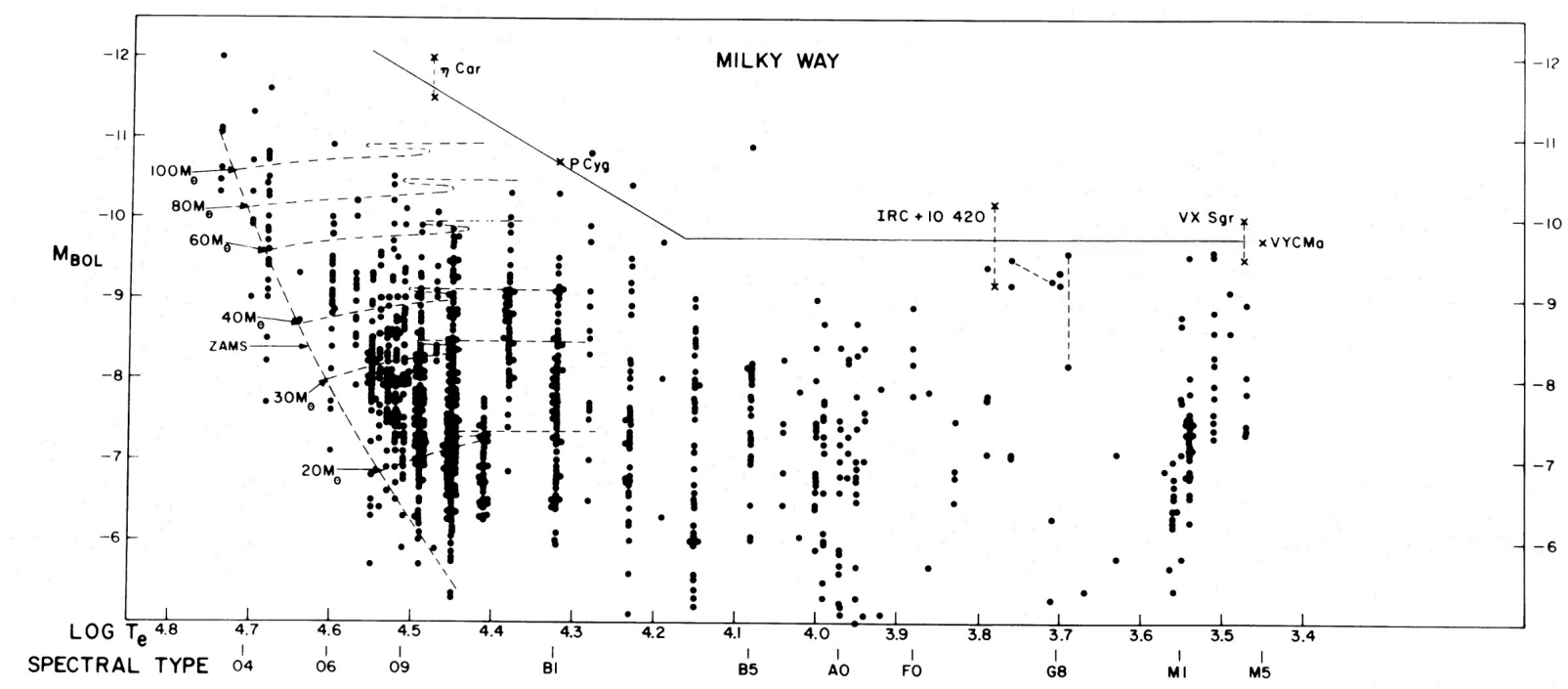

FIG. 3.-The "theoretical" H-R diagram, $M_{\text {bol }}$ versus $\log T_{e}$, for the galactic supergiants. The position of the ZAMS and evolutionary tracks with mass loss are shown. The solid line defines the approximate upper boundary of the supergiant luminosities. The positions of two peculiar stars, $\eta$ Car and $P$ Cyg, and three supergiant infrared sources are also
indicated.

Figure 2. The HR Diagram, $\mathrm{M}_{B o l}$ vs. $\log \mathrm{T}$, for the luminous stars in the Milky Way from [25].

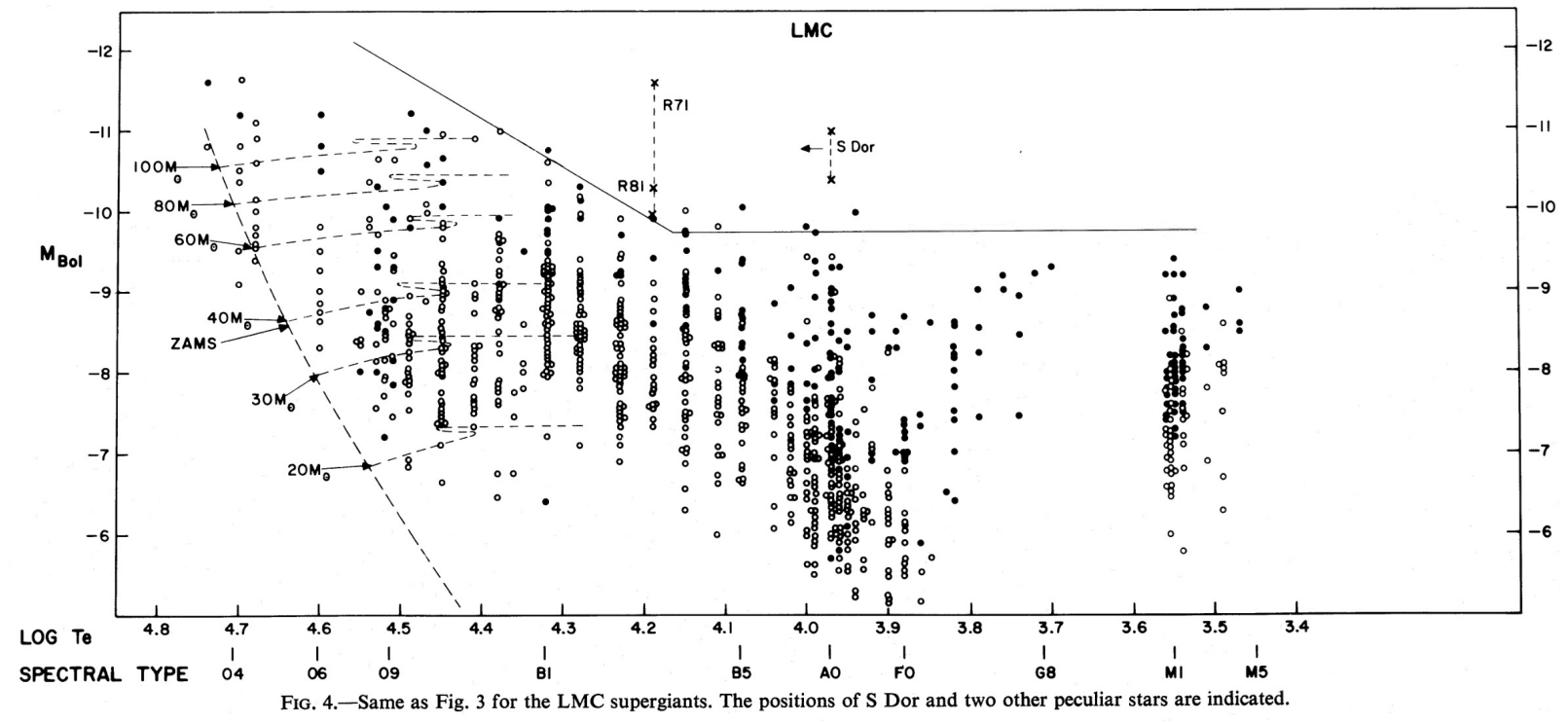

Figure 3. The HR Diagram, $\mathrm{M}_{B o l}$ vs. $\log \mathrm{T}$, for the luminous stars in the LMC from [25].

This empirical boundary is often referred to in the literature as the "Humphreys-Davidson" Limit. It was not predicted by theory or the stellar structure models and evolutionary tracks at that time. The lack of evolved stars, post main sequence stars, above a certain luminosity implies an upper limit to the masses of stars that can evolve to become red supergiants thus altering the previously expected evolution of the most massive stars across the HR Diagram. In the original study, this limit corresponded to an initial mass near $60 \mathrm{M}_{\odot}$. Improved models with mass loss and rotation suggest a mass more like $40-50 \mathrm{M}_{\odot}$ today.

Spectroscopy of luminous star candidates in other Local Group galaxies confirmed the upper luminosity boundary in galaxies of different types, but, in most cases, surveys and population studies were minimal: M31 [26], IC 1613 and NGC 6822 [27]. Humphreys \& Sandage [28] completed a major survey for the brightest blue and red stars in M33, that provided the basis for spectroscopy and identification of the most luminous supergiants in that nearby spiral [29], and in subsequent studies. Similar HR Diagrams for 
the massive stars in the SMC were published a few years later by Humphreys [30] based on a combination of previous work and new observations.

At the time the Humphreys-Davidson paper was published, it was apparent that significant mass loss occurred in both the blue and red supergiants and there was increasing interest in the role that mass loss may have on their evolution. The lack of evolved cooler counterparts to the most massive evolved hot stars in both galaxies suggested that their post main sequence evolution was of special interest. A few high luminosity stars were known for their instabilities, variability and evidence for high mass loss such as eta Car and P Cyg in the Milky Way and S Dor in the LMC. Several luminous blue variables, spectroscopically similar to eta Car and P Cyg, were now recognized in other galaxies [31,32]. As a group, they were known as S Doradus Variables or as Hubble-Sandage Variables in M31 and M33. Several of these stars were included on the HR Diagrams. They were increasing evidence that the phenomena of high mass loss and instabilities, as observed in eta Car and P Cyg, were more common. Humphreys and Davidson thus suggested that the most massive hot stars could not evolve to cooler temperatures because of their instabilities resulting in high mass loss. The temperature dependence of the luminosity limit for the hottest stars was evidence that the instability was mass dependent. This mass loss could be unsteady and much greater at times resulting in high mass loss events. The relatively tight upper luminosity boundary for the cooler stars represented the upper limit to the initial masses of stars that could evolve across the HR Diagram in a stable way to become red supergiants (RSGs).

Some of the first evolutionary tracks with mass loss were also being published at that time [33-36]. It was shown that high mass loss (higher than observed) would cause the tracks to reverse and the stars evolve back to warmer temperatures.

\section{Surveys and More Surveys}

We emphasize the importance of spectroscopy for the identification and analysis of the most luminous stars, but surveys are essential for identifying candidates. Beginning in the 1980s, astronomers began to add multi-wavelength surveys, primarily in the near-infrared, to complement the traditional optical photometry. Elias, Frogel, \& Humphreys [37] obtained near IR photometry for known and candidate red supergiants in the LMC and SMC for a comparison with the Galactic population. The all-sky near-infrared 2MASS survey from 1.2 to $2.2 \mu \mathrm{m}$ [38] reached the brightest stars in M31 and M33 as well as the Clouds. The infrared observations allowed astronomers to look for free-free emission from the stellar winds and the presence of circumstellar dust, another indicator of mass loss, and to correct the luminosites for possible additional extinction due to circumstellar dust.

Space-based telescopes such as UIT and later GALEX added FUV and NUV imaging and photometry for more complete SEDS at the shorter wavelengths and more accurate estimates of the total luminosities for the hottest supergiants. The mid-infrared surveys with Spitzer/IRAC of the Magellanic Clouds [39,40] and M31 [41] and M33 [42] added fluxes from 3 to $8 \mu \mathrm{m}$ and even longer wavelengths to search for colder dust, thus allowing us to investigate their mass loss histories.

With the advent of the wide-field CCD mosaic cameras, ground-based, multi-wavelength optical surveys, such as the Local Group (LGGS) by Massey et al. [43], added to the fundamental data. These ground-based surveys however are seeing-limited and lack spatial resolution, thus the images are often multiple. Here, again, spectroscopy or higher resolution imaging with Adaptive Optics on large telescopes can identify and even separate the stars. These ground-based surveys are enhanced by observations with the Hubble Space Telescope such as the PHAT surveys in M31 and M33 [44]. Numerous imaging programs of other galaxies intended for other purposes with HST have provided lists of resolved stars for further observation in, for example, M101 [45] and NGC 2403 and M81 [46,47]. 


\section{Stellar Population Comparisons}

One of the outstanding stellar evolution questions is how stellar populations may depend on their environment, namely the properties of the host galaxy, its mass and luminosity, the fraction of interstellar gas and dust, and especially on the chemical composition or metallicity. As the most luminous and visually brightest stars, massive stars provide the first indicator or measurement of how star formation, evolution, and the terminal state, depend on these factors. Mass loss is known to alter stellar evolution, and in the standard picture of line-driven winds from hot stars, mass loss is also expected to be metallicity dependent and significantly less in lower metallicity systems.

The first evidence for significant differences in the properties of the massive star populations was the well-established absolute magnitude dependence of the visually "brightest blue star" on the galaxy type or luminosity in the surveys for extragalactic distance indicators [14]. The smaller, less massive galaxies had fewer of the most massive stars, thus their evolved counterparts were statistically less likely to become the most luminous observed blue stars. These results suggest that the star formation rate for these most massive stars was less in the smaller galaxies. In contrast, the luminosities of the brightest red stars showed little or no dependence on the host galaxy. The stars of somewhat lower initial mass thus existed in sufficient numbers in the smaller galaxies to produce evolved descendants in the red supergiant region, and the luminosities of their brightest members reflected the upper luminosity boundary.

The metallicity dependence of the overall characteristics of the massive star populations in different galaxies has been less apparent. Comparison of the HR Diagrams of the massive stars in our region of the Milky Way with the LMC revealed very similar populations [25]. The LMC oxygen abundance is lower than Solar but by no more than a factor of two. Consequently, a comparison with the outer regions of the Milky Way, also with reduced metallicity, not surprisingly, showed little variation. Among our Local Group galaxies with comprehensive stellar surveys for luminous stars, the SMC has the lowest metallicity, about $1 / 10$ th Solar, and a reduced oxygen abundance by about a factor of five.

Early tell-tale evidence for an observable metallicity affect was the distribution of the spectral types of the M supergiants [48]. A preliminary survey of red supergiants in the LMC and SMC compared to known Galactic RSGs, revealed a dramatic shift in their spectral types in the SMC to much earlier spectral types, compared to the LMC and Milky Way stars. Except for one M2 -type star in the SMC, all of the others were type $\mathrm{M} 0$ or earlier. This apparent shift, attributed to weaker TiO bands, was due to the lower SMC metallicity resulting in lower opacities in the atmospheres. The spectra thus arise in warmer layers. This result was confirmed in later surveys that extended to fainter magnitudes [37,49].

Stellar wind theory predicts a measurable dependence of the mass loss rate on metallicity [50], decreasing with declining heavy element abundances, but the measured rates [51] are somewhat higher than expected. Clumping in the stellar winds is another complication which when included in the mass loss models reduces the mass loss rates [52-54]. Measurement of the stellar wind properties and mass loss rates in the luminous, hot OB-type stars in nearby galaxies, especially in the Magellanic Clouds, has progressed with the advent of very large telescopes equipped with high resolution spectrographs. Paul Crowther's article in this issue on the FLAMES survey of the luminous, hot stars in the Clouds discusses their winds and mass loss rates.

\section{The Most Luminous Stars of Different Types}

Since the early work of the 1970s and 1980s to identify the most luminous and brightest stars in nearby galaxies, numerous surveys and studies of the massive stars, primarily in Local Group galaxies, have greatly expanded the completeness of the population samples. These include, in the LMC and SMC, surveys for the yellow and red supergiants [55,56], and, in M31 and M33, studies of the luminous star population [57-61] and surveys for the yellow and red supergiants [62-66]. Surveys and follow-up 
spectroscopy of the luminous blue and red stars in the Local Group irregulars NGC 6822 and IC 1613 are less complete $[27,67-69]$, but they provide an additional sample of the massive stars in two smaller galaxies and also with reduced metallicity.

Table 1 presents a summary of the most luminous stars of different spectral types or temperature ranges in six Local Group Galaxies with their morphological types and integrated visual luminosities. Initial samples of the massive stars in the well-studied nearby spirals, M101, M81 and NGC 2403, outside our Local Group, have also been observed [45-47,70-72] and are included here. The bolometric luminosities of the stars are listed for the three highest in each spectral type group for each galaxy. The adopted distance moduli (Table 2) were used to determine the luminosities. The O-type stars are not included because many are eventually recognized as binary or are in multiple systems. Likewise, those stars in extremely crowded fields, including many OB-type stars, are not listed since they may be blended. Consequently, this table does not include those stars that will be the intrinsically most luminous, most massive members of their home galaxies. A few stars of special interest are identified by name in the table. Some of them are discussed in other articles in this issue. Note that the Galactic stars are not included. The upper HR Diagram for the Milky Way needs to re-examined when the Gaia survey is complete with improved distances and very likely with a larger volume sample.

Table 1. The Most Luminous Stars $\left(\mathrm{M}_{B o l}\right)$ of Different Spectral Types.

\begin{tabular}{|c|c|c|c|c|c|c|c|c|c|}
\hline $\begin{array}{l}\text { Galaxy } \\
\text { Type } \\
\mathbf{M}_{v}\end{array}$ & $\begin{array}{l}\text { M31 } \\
\text { Sb I-II } \\
-21.5\end{array}$ & $\begin{array}{l}\text { M33 } \\
\text { Sc II } \\
-18.9 \\
\end{array}$ & $\begin{array}{l}\text { LMC } \\
\text { Im } \\
-18.5 \\
\end{array}$ & $\begin{array}{l}\text { SMC } \\
\text { Im } \\
-16.8 \\
\end{array}$ & $\begin{array}{l}\text { NGC } 6822 \\
\text { Im } \\
-15.2\end{array}$ & $\begin{array}{l}\text { IC } 1613 \\
\text { Ir } \\
-14.8\end{array}$ & $\begin{array}{l}\text { NGC } 2403 \\
\text { Sc III } \\
-19.3 \\
\end{array}$ & $\begin{array}{l}\text { M81 } \\
\text { Sb I-II } \\
-20.9 \\
\end{array}$ & $\begin{array}{l}\text { M101 } \\
\text { Sc I } \\
-21.0 \\
\end{array}$ \\
\hline $\begin{array}{l}\text { Spectral Type } \\
\text { O9.5-B5 }\end{array}$ & $\begin{array}{l}\mathrm{M}_{\text {Bol }} \\
-10.6(2) \\
-10.2 \\
-10.0(2)\end{array}$ & $\begin{array}{l}\mathrm{M}_{\text {Bol }} \\
-10.1 \\
-10.0 \\
-9.8 \\
\end{array}$ & $\begin{array}{l}\mathrm{M}_{\text {Bol }} \\
-10.9 \\
-10.7 \\
-10.0 \\
\end{array}$ & $\begin{array}{l}\mathrm{M}_{\text {Bol }} \\
-10.3(2) \\
-10.2 \\
-9.8(2)\end{array}$ & $\begin{array}{l}\mathrm{M}_{\text {Bol }} \\
-10.3 \\
-9.9 \\
-9.5 \\
\end{array}$ & $\begin{array}{l}\mathrm{M}_{\text {Bol }} \\
-9.5 \\
-8.8 \\
-8.7\end{array}$ & $\begin{array}{l}\mathrm{M}_{\text {Bol }} \\
-10.0(2) \\
-9.5 \\
-9.4\end{array}$ & $\begin{array}{l}\mathrm{M}_{\text {Bol }} \\
-10.6 \\
-10.0 \\
-9.7 \\
\end{array}$ & $\begin{array}{l}\mathrm{M}_{\text {Bol }} \\
\ldots \\
\ldots \\
\ldots \\
\end{array}$ \\
\hline B8-A8 & $\begin{array}{l}-9.3 \\
-9.1 \\
-8.9(2)\end{array}$ & $\begin{array}{l}-10.1(\mathrm{~B} 324) \\
-9.5(2) \\
-9.4\end{array}$ & $\begin{array}{l}-9.6(\mathrm{HD} 33579) \\
-9.0 \\
-8.9\end{array}$ & $\begin{array}{l}-9.6(\mathrm{HD} 7583) \\
-9.2 \\
-9.0\end{array}$ & $\begin{array}{l}-8.1 \\
-7.7 \\
-7.5\end{array}$ & $\begin{array}{l}-8.4 \\
-6.9 \\
-6.7\end{array}$ & $\begin{array}{l}-9.8 \\
-9.5 \\
-9.4\end{array}$ & $\begin{array}{l}-9.3 \\
-9.1(2) \\
-8.9\end{array}$ & $\begin{array}{l}-10.4(2) \\
-10.3 \\
-9.9\end{array}$ \\
\hline FGK & $\begin{array}{l}-9.8 \\
-9.7 \\
-9.5\end{array}$ & $\begin{array}{l}-9.6(2) \\
-9.5(\operatorname{Var} A) \\
-9.2(3)\end{array}$ & $\begin{array}{l}-9.3 \\
-9.2 \\
-8.9(3)\end{array}$ & $\begin{array}{l}-9.2 \\
-9.1 \\
-8.1\end{array}$ & $\begin{array}{l}\cdots \\
\cdots \\
\cdots\end{array}$ & $\begin{array}{l}\cdots \\
\cdots \\
\cdots\end{array}$ & $\begin{array}{l}-9.3 \\
-8.8 \\
-8.5\end{array}$ & $\begin{array}{l}-9.7 \\
-9.6 \\
-9.4\end{array}$ & $\begin{array}{l}-9.8 \\
-9.3 \\
-9.2\end{array}$ \\
\hline RSGs & $\begin{array}{l}-9.4(2) \\
-9.3 \\
-9.1(2)\end{array}$ & $\begin{array}{l}-9.6 \\
-9.5(2) \\
-9.4(2)\end{array}$ & $\begin{array}{l}-9.6(\mathrm{MOH}-\mathrm{G} 64) \\
-9.1 \\
-9.0\end{array}$ & $\begin{array}{l}-9.2 \\
-9.1 \\
-8.9\end{array}$ & $\begin{array}{l}-9.2 \\
-9.1 \\
-8.7\end{array}$ & $\begin{array}{l}-9.4 \\
-8.8 \\
-8.7\end{array}$ & $\begin{array}{l}-8.5 \\
-8.4(2) \\
\ldots\end{array}$ & $\begin{array}{l}-9.5: \\
-9.2(2) \\
\ldots\end{array}$ & $\begin{array}{l}-9.9: \\
-9.5 \\
-9.2\end{array}$ \\
\hline
\end{tabular}

Some comments with respect to the data in this table are helpful. It is clear that data for some of the spectral type groups are lacking or incomplete such as for the YSGs (FGK) in NGC 6822 and IC 1613. Those stars are undoubtedly present, but have just not been identified in the published surveys, and they may also be of somewhat lower luminosity in those two galaxies. A survey to identify and classify the hotter supergiants in M101 has not been completed, and, although a survey identifying RSGs in NGC 2403 exists [72], confirming spectroscopy and photometry is lacking. In general, the numbers for the hot supergiant group (O9.5-B5) may not include the most luminous members because many of these stars are in crowded regions.

The six Local Group galaxies in Table 1 present a diverse group of galaxy types with a wide range of luminosities. The well known dependence of the most luminous "blue" stars on the parent galaxy is especially notable for the evolved, post-main sequence A-type supergiants in the two lowest luminosity and lowest mass galaxies, NGC 6822 and IC 1613. Together with the SMC, these are also the galaxies with the lowest metallicity, but the heavy element abundance in NGC 6822, however, is intermediate between the SMC and LMC. Reduced metallicity was also expected to reduce the opacity in the stellar atmospheres and increase their absolute visual magnitudes but the data for these galaxies show the opposite effect. 
Thus, although metallicity undoubtedly plays a role in stellar evolution, the luminosity and mass of the parent galaxy is the primary determinant, to the first order, for the luminosities of the most luminous stars. This is a size of sample effect. Assuming a similar slope for the mass function, the most massive galaxies will have a larger progenitor population of massive stars, and consequently, at any given time, we will therefore be more likely to observe their most luminous, evolved counterparts.

We also see another effect with this group of galaxies related to the galaxy type. M31 (Sb I-II) and M81 (Sb I-II), both high luminosity spirals, have lower luminosity evolved A-type blue stars compared with the Sc-type spirals and Magellanic irregulars. This second effect very likely reflects a dependence on the lower star formation rate, not mass, in the Sb spirals. Both of these spirals have above solar metallicites, although M81 has a metallicity gradient in its disk similar to the Milky Way and the Sc spirals M33 and M101, and solar-type abundances in the outer parts.

This degeneracy, wherein the luminosity of the most luminous blue stars are dependent on the host galaxy, complicated their use as direct distance indicators. The most luminous red supergiants, however, exhibit a nearly constant upper luminosity, related to the Humphreys-Davidson limit, over a wide range of galaxy types and luminosities. The presence of extensive circumstellar dust and uncertain reddening in the most luminous RSGs though limits their usefulness as distance indicators in the visual, but their luminosities in the infrared need to be further studied. Kudritzki [73] introduced a new distance determination method for B- and A-type supergiants, the Flux-weighted Gravity-Luminosity Relation (FGLR), that depends on quantitative analysis of the Balmer lines measured in low resolution spectra with good $\mathrm{S} / \mathrm{N}$ ratio. It has been successfully applied to several nearby galaxies $[47,74,75]$, demonstrating that the visually brightest stars, the A-type supergiants, have potential as distance indicators at very large distances.

\section{The Complex Upper HR Diagram}

The upper luminosity boundary to the HR Diagram, or Humphreys-Davidson Limit, complicates our understanding of massive star evolution. Above some initial mass, $\approx 40-50 \mathrm{M}_{\odot}$, the stars do not evolve across the HR Diagram to become RSGs. Their evolution to cooler temperatures is most likely halted by proximity to the modified Eddington Limit and the accompanying high mass loss episodes. The opacity "Modified Eddington Limit", well-known since the mid-1980's [76], see the papers and discussion in [77,78], describes an instability that arises when $L / M$ approaches the classical $L / M_{E d d}$ value [79]. Models based on this and other instabilities have been proposed to reproduce the upper limit in the HR Diagram [80-82]. See Humphreys \& Davidson [83] for a review.

The stars then evolve back to warmer temperatures. Having shed a lot of mass, they are now increasingly subject to atmospheric instabilities before their terminal state. They thus have a different evolutionary path and different mass loss histories than their somewhat lower mass counterparts in the same galaxy or even the same cluster. These lower mass supergiants, from $\approx 9$ or 10 up to $30-40 \mathrm{M}_{\odot}$ or so, evolve across the HR Diagram, becoming red supergiants which alters their interior structure and with enhanced mass loss. Some may also evolve back to a warmer state, perhaps as warm hypergiants, to become LBVs, or B[e] supergiants, where their final fate is most likely as core-collapse supernovae.

Consequently, the upper HR Diagram is populated by a diversity of evolved, luminous and variable stars of different types that challenge our understanding of their physics, evolution and eventual fate. Many of them are distinguished by their emission line spectra, and evidence for stellar winds and mass loss. In addition, some of them exhibit periods of enhanced mass loss, such as the LBVs/S Dor variables and the warm and cool hypergiants with their resolved ejecta. In addition, Wolf-Rayet stars of various types, Oe and Of stars, the B[e] supergiants, and the Fe II emission line stars occupy the same parts of the HR Diagram. They may or may not be related. They could be stars of similar initial mass but in different 
stages of their evolution or have experienced different mass loss histories, and some may be binaries. This diversity is one of the challenges to understanding massive stars, their evolution, and eventual fate.

The HR Diagrams below (Figure 4) for M31 and M33 show the distribution in the luminositytemperature plane of three of these mass losing classes of stars, the LBVs, $\mathrm{B}[\mathrm{e}]$ supergiants and the warm hypergiants.

One of the outstanding questions is the final fate of the most massive stars. It used to be simply assumed that all stars much above initial masses of $10 \mathrm{M}_{\odot}$ or so, would end their brief lives as some kind of supernova, but their final stage as core-collapse SNe is now in question. Smartt $[84,85]$ suggested an upper mass limit of $\approx 18 \mathrm{M}_{\odot}$ for the red supergiant progenitors of the Type II-P SNe, while Jennings et al. [86] found a lack of massive supernova progenitors in M31 and M33 and suggested an upper mass of 35-45 $\mathrm{M}_{\odot}$. Do the most massive stars collapse directly to black holes instead, long suspected for extreme, very massive stars such as eta Carinae?
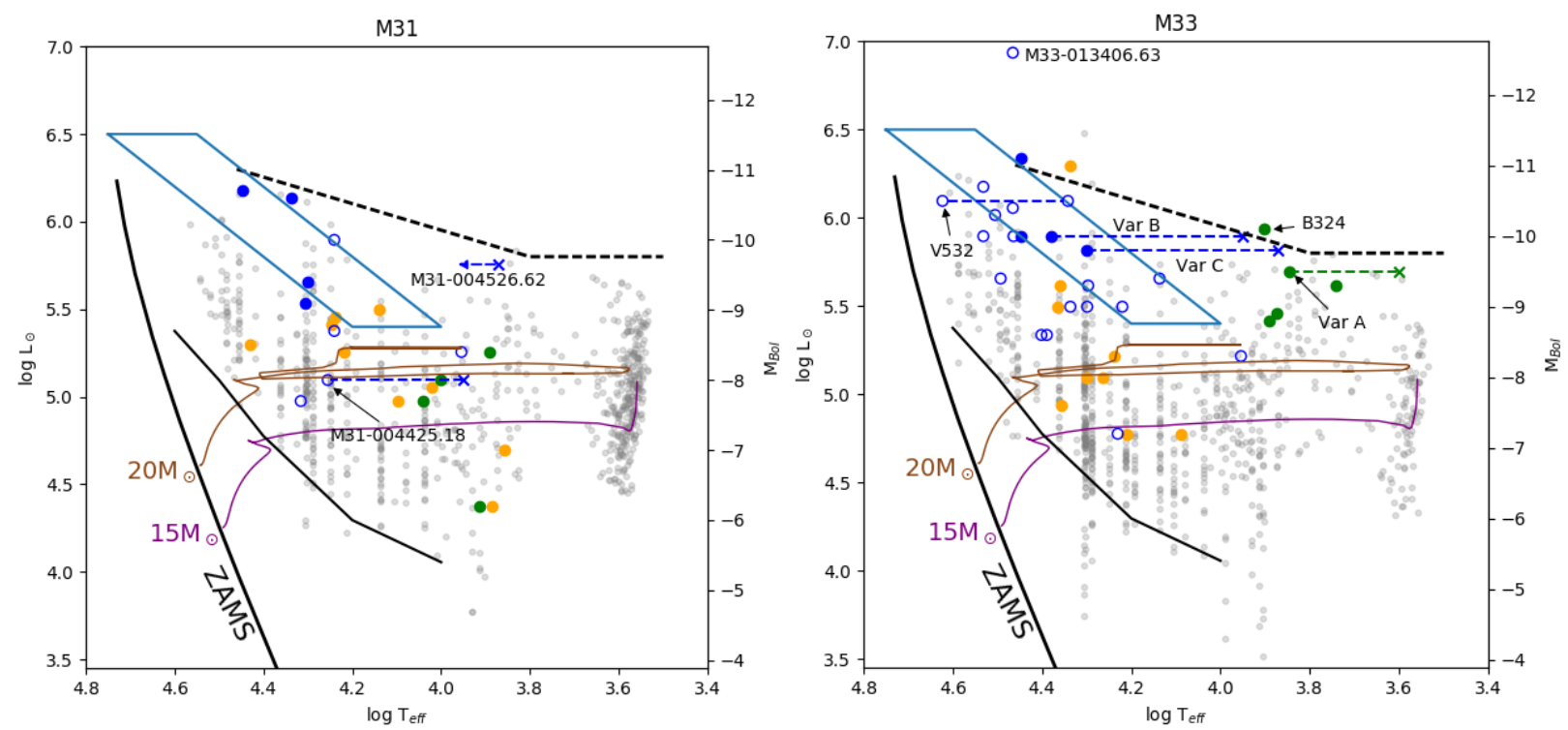

Figure 4. The schematic HR Diagrams for M31 and M33 showing the positions of the confirmed LBVs and candidate LBVs shown respectively, as filled and open blue circles, the warm hypergiants as green circles and the $\mathrm{B}[\mathrm{e}]$ supergiants as orange circles. The LBV transits during their high mass loss state are shown as dashed blue lines. The LBV/S Dor instability strip is outlined in blue. The 15 and $20 \mathrm{M}_{\odot}$ tracks are from [87] with rotation are shown to provide a reference for the lower mass B[e]sgs, which are probable rotators. (Higher mass tracks are not shown due to crowding.) The supergiant population is shown in the background in light gray. Reproduced from [61].

In addition, the supernova surveys have identified numerous non-terminal giant eruptions, in which the object greatly increases its total luminosity possibly expelling several solar masses and the star survives. Some of these events are confused with true SNe and thus have been called "supernova impostors". This is a diverse group of objects with a range of luminosities and possible progenitors. A few impostors appear to be normal LBV/S Dor variables in their eruptive or maximum light state. Most are giant eruptions, possibly similar to eta Car [83] from evolved massive stars, while some are red transients (ILRTs [88]) from a lower mass population. The origin of the instability in these giant eruptions is unknown, but proximity to the Eddington Limit is crucial [89]. It is not known what role these high mass loss events may play in the final stages of massive star evolution or their relation to the evolved massive star population and to other stars with instabilities such as the LBVs and the warm hypergiants. 
Thus, we observe a complex upper HR Diagram with different evolutionary paths dependent on initial mass, and several types of evolved stars not only experiencing continuous mass loss, but also high mass loss events. The study of luminous stars in the nearer galaxies provides us with an improved census of these evolved stars, their relative numbers, physical properties and behavior, and clues to their evolutionary state and possible relationship to each other on the HR Diagram. In this Special Issue on luminous stars in nearby galaxies, the reviews focus on different examples of evolved massive stars; the luminous $\mathrm{O}$ and B-type stars in the Magellanic Clouds, LBVs, B[e] supergiants, the warm hypergiants, and the Wolf-Rayet stars.

Although the articles are about different types of evolved massive stars, many are in the same galaxies. We have therefore adopted the following distance moduli based on the Cepheid scale for these nearby galaxies for consistency and for ease of cross-referencing and comparison.

Table 2. Adopted Distance Moduli.

\begin{tabular}{ccc}
\hline Galaxy & Distance Modulus (mag) & Comment \\
\hline LMC & 18.5 & Cepheids \\
SMC & 18.9 & Cepheids \\
M31 & 24.4 & Cepheids [90] \\
M33 & 24.5 & Cepheids [91] \\
NGC 6822 & 23.4 & Cepheids [92] \\
IC 1613 & 24.3 & Cepheids [93] \\
NGC 2403 & 27.5 & Cepheids [94] \\
M81 & 27.8 & Cepheids [94] \\
M101 & 29.1 & Cepheids [94] \\
\hline
\end{tabular}

Funding: This research received no external funding.

Conflicts of Interest: The authors declare no conflict of interest.

\section{References}

1. Tammann, G.A.; Sandage, A. The stellar content and distance of the galaxy NGC 2403 in the M81 group. Astrophys. J. 1968, 151, 825-860. [CrossRef]

2. Hubble, E.; Sandage, A. The brightest variable stars in extragalactic nebulae. I. M31 and M33. Astrophys. J. 1953, 118, 353-361. [CrossRef]

3. Cannon, A.J. The Henry Draper extension. Ann. Astron. Obs. Harvard Coll. 1936, 100, 367.

4. Cannon, A.J.; Pickering, E.C. The Henry Draper catalog. Ann. Astron. Obs. Harvard Coll. 1924, 91-99.

5. Feast, M.W.; Thackeray, A.D.; Wesselink, A.J. The brightest stars in the Magellanic Clouds. Mon. Not. R. Astron. Soc. 1960, 121, 337-385. [CrossRef]

6. Henize, K. Catalogues of $\mathrm{H} \alpha$-emission Stars and Nebulae in the Magellanic Clouds. Astrophys. J. 1956, 2, 315-344. [CrossRef]

7. Sanduleak, N. A finding list of proven or probable Small Magellanic Clouds members. Astron. J. 1968, 73, 246-250. [CrossRef]

8. Sandulaeak, N. A Deep Objective-Prism Survey for Large Magellanic Cloud Members; The Cerro Tololo Inter-American Observatory: Coquimbo Region, Chile, 1970.

9. Fehrenbach, C.; Duflot, M. Large Magellanic Cloud. List of LMC members and lis t of galactic stars. Charts for recognizing these stars on the maps published by the Smithsonian Institution Astrophysical Observatory. Astron. Astrophys. Suppl. 1970, 10, 231.

10. Westerlund, B. An infrared survey of the Magellanic Clouds. I. Four regions in the Large Cloud. Uppsala Astron Obs. Ann. 1960, 4, 7.

11. Westerlund, B. Population I in the Large Magellanic Cloud. Astron. J. 1961, 5, 1-28. 
12. Blanco, V.M.; McCarthy, M.F.; Blanco, B. Carbon and late M-type stars in the Magellanic Clouds. Astrophys. J. 1980, 242, 938-964. [CrossRef]

13. Blanco, V.M.; McCarthy, M.F. The distribution of carbon and M-type giants in the Magellanic Clouds. Astron. J. 1983, 88, 1442-1457. [CrossRef]

14. Sandage, A.; Tammann, G.A. Steps toward the Hubble constant. II. The brightest stars in late-type spiral galaxies. Astrophys. J. 1974, 191, 603-621. [CrossRef]

15. Humphreys, R.M. Studies of luminous stars in nearby galaxies. I. Supergiants and O stars in the Milky Way. Astrophys. J. 1978, 38, 309-350. [CrossRef]

16. Humphreys, R.M. Spectroscopic and photometric observations of luminous stars in Carina-Centaurus (l=282d-305d). Astron. Astrophys. 1973, 9, 85-96.

17. Humphreys, R.M. Spectroscopic and photometric observations of luminous stars in the Centaurus-Norma $(1=305-340)$ section of the Milky Way. Astron. Astrophys. Suppl. 1975, 19, 243-247.

18. Walborn, N.R. Spectral classification of OB stars in both hemispheres and the absolute-magnitude calibration. Astron. J. 1972, 77, 312-318. [CrossRef]

19. Humphreys, R.M.; Strecker, D.W.; Ney, E.P. Spectroscopic and Photometric Observations of M Supergiants in Carina. Astrophys. J. 1972, 172, 75-88. [CrossRef]

20. Humphreys, R.M.; Ney, E.P. Visual and infrared observations of late-type supergiants in the southern sky. Astrophys. J. 1974, 194, 623-628. [CrossRef]

21. Humphreys, R.M. Studies of luminous stars in nearby galaxies. II-M supergiants in the Large Magellanic Cloud. Astrophys. J. 1979, 39, 389-403. [CrossRef]

22. Ardeberg, A.; Brunet, J.P.; Maurice, E.; Prevot, L. Spectrographic and photometric observations of supergiants and foreground stars in the direction of the Large Magellanic Cloud. Astron. Astrophys. Suppl. 1972, 6, $249-309$.

23. Brunet, J.-P.; Imbert, M.; Martin, N.; Mianes, P.; Prévot, L.; Rebeirot, E.; Rousseau, J. Studies of the LMC stellar content. I. A catalogue of 272 new O-B2 stars. Astron. Astrophys. Suppl. 1975, 21, 109-136.

24. Walborn, N.R. Spectral classification of O and B0 supergiants in the Magellanic Clouds. Astrophys. J. 1977, 215, 53-57. [CrossRef]

25. Humphreys, R.M.; Davidson, K. Studies of luminous stars in nearby galaxies. III-Comments on the evolution of the most massive stars in the Milky Way and the Large Magellanic Cloud. Astrophys. J. 1979, 232, 409-420. [CrossRef]

26. Humphreys, R.M. Studies of luminous stars in nearby galaxies. IV-Baade's field IV in M31. Astrophys. J. 1979, 234, 854-860. [CrossRef]

27. Humphreys, R.M. Studies of luminous stars in nearby galaxies. V-The local group irregulars NGC 6822 and IC 1613. Astrophys. J. 1980, 238, 65-78. 086/157958. [CrossRef]

28. Humphreys, R.M.; Sandage, A. On the stellar content and structure of the spiral Galaxy M33. Astrophys. J. 1980, 44, 319-381. [CrossRef]

29. Humphreys, R.M. Studies of luminous stars in nearby galaxies. VI-The brightest supergiants and the distance to M33. Astrophys. J. 1980, 241, 587-597. [CrossRef]

30. Humphreys, R.M. Studies of luminous stars in nearby galaxies. VIII-The Small Magellanic Cloud. Astrophys. J. 1983, 265, 176-193. [CrossRef]

31. Humphreys, R.M. The spectra of AE Andromedae and the Hubble-Sandage variables in M31 and M33. Astrophys. J. 1975, 200, 426-429. [CrossRef]

32. Humphreys, R.M. Luminous variable stars in M31 and M33. Astrophys. J. 1978, 219, 445-451. [CrossRef]

33. Chiosi, C.; Nasi, E.; Sreenivasan, S.R. Massive stars evolution with mass-loss. Astron. Astrophys. 1978, 63, $103-124$.

34. De Loore, C.; De Greve, J.P.; Lamers, H.J.G.L.M. Evolution of massive stars with mass loss by stellar wind. Astron. Astrophys. 1977, 61, 251-259.

35. Maeder, A. The most massive stars in the Galaxy and the LMC-Quasi-homogeneous evolution, time-averaged mass loss rates and mass limits. Astron. Astrophys. 1980, 92, 101-110.

36. Maeder, A. Grids of evolutionary models for the upper part of the HR diagram. Mass loss and the turning of some red supergiants into WR stars. Astron. Astrophys. 1981, 102, 401-410. 
37. Elias, J.H.; Frogel, J.A.; Humphreys, R.M. M supergiants in the Milky Way and the Magellanic Clouds: Colors, spectral types, and luminosities. Astrophys. J. 1985, 57, 57-131. [CrossRef]

38. Skrutskie, M.F.; Cutri, R.M.; Stiening, R.; Weinberg, M.D.; Schneider, S.; Carpenter, J.M.; Beichman, C.; Capps, R.; Chester, T.; Elias, J.; et al. The Two Micron All Sky Survey (2MASS). Astron. J. 2006, 131, 1163-1183. [CrossRef]

39. Bonanos, A.; Massa, D.L.; Sewilo, M.; Lennon, D.J.; Panagia, N.; Smith, L.J.; Meixner, M.; Babler, B.L.; Bracker, S.; Meade, M.R.; et al. Spitzer SAGE Infrared Photometry of Massive Stars in the Large Magellanic Cloud. Astron. J. 2009, 138, 1003-1021. -6256/138/4/1003. [CrossRef]

40. Bonanos, A.; Lennon, D.J.; Köhlinger, F.; Van Loon, J.T.; Massa, D.L.; Sewilo, M.; Evans, C.J.; Panagia, N.; Babler, B.L.; Block, M.; et al. Spitzer SAGE-SMC Infrared Photometry of Massive Stars in the Small Magellanic Cloud. Astron. J. 2010, 140, 416-429. 6256/140/2/416. [CrossRef]

41. Mould, J.; Barmby, P.; Gordon, K.; Willner, S.P.; Ashby, M.L.N.; Gehrz, R.D.; Humphreys, R.; Woodward, C.E. A Point-Source Survey of M31 with the Spitzer Space Telescope. Astrophys. J. 2008, 687, 230-241. [CrossRef]

42. McQuinn, K.B.W.; Woodward, C.E.; Willner, S.P.; Polomski, E.F.; Gehrz, R.D.; Humphreys, R.M.; van Loon, J.T.; Ashby, M.L.N.; Eicher, K.; Fazio, G.G. The M33 Variable Star Population Revealed by Spitzer. Astrophys. J. 2007, 664, 850-861. [CrossRef]

43. Massey, P.; Olsen, K.A.G.; Hodge, P.W.; Strong, S.B.; Jacoby, G.H.; Schlingman, W.; Smith, R.C. A Survey of Local Group Galaxies Currently Forming Stars. I. UBVRI Photometry of Stars in M31 and M33. Astron. J. 2006, 131, 2478-2496. [CrossRef]

44. Dalcanton, J.J.; Williams, B.F.; Lang, D.; Lauer, T.R.; Kalirai, J.S.; Seth, A.C.; Dolphin, A.; Rosenfield, P.; Weisz, D.R.; Bell, E.F.; et al. The Panchromatic Hubble Andromeda Treasury. Astrophys. J. 2012, 200, 1-37. [CrossRef]

45. Grammer, S.H.; Humphreys, R.M.; Gerke, J. The Massive Star Population in M101. III. Spectra and Photometry of the Luminous and Variable Stars. Astron. J. 2015, 149, 1-15. [CrossRef]

46. Humphreys, R.M.; Stangl, S.; Gordon, M.S.; Davidson, K.; Grammer, S.H. Luminous and Variable Stars in NGC 2403 and M81. Astron. J. 2019, 157, 1-16. [CrossRef]

47. Kudritzki, R.-P.; Urbaneja, M.A.; Gazak, Z.; Bresolin, F.; Przybilla, N.; Gieren, W.; Pietrzyński, G. Quantitative Spectroscopy of Blue Supergiant Stars in the Disk of M81: Metallicity, Metallicity Gradient, and Distance. Astrophys. J. 2012, 747, 15. [CrossRef]

48. Humphreys, R.M. M supergiants and the low metal abundances in the Small Magellanic Cloud. Astrophys. J. 1979, 231, 384-389. [CrossRef]

49. Massey, P.; Olsen, K.A.G. The Evolution of Massive Stars. I. Red Supergiants in the Magellanic Clouds. Astron. J. 2003, 126, 2867-2886. [CrossRef]

50. Vink, J.S.; de Koter, A.; Lamers, H.J.G.L.M. Mass-loss predictions for O and B stars as a function of metallicity. Astron. Astrophys. 2001, 369, 574-588.:20010127. [CrossRef]

51. Mokiem, M.R.; de Koter, A.; Vink, J.S.; Puls, J.; Evans, C.J.; Smartt, S.J.; Crowther, P.A.; Herrero, A.; Langer, N.; Lennon, D.J.; et al. The empirical metallicity dependence of the mass-loss rate of O- and early B-type stars. Astron. Astrophys. 2007, 473, 603-614.:20077545. [CrossRef]

52. Crowther, P.A.; Hillier, D.J.; Evans, C.J.; Fullerton, A.W.; De Marco, O.; Willis, A.J. Revised Stellar Temperatures for Magellanic Cloud O Supergiants from Far Ultraviolet Spectroscopic Explorer and Very Large Telescope UV-Visual Echelle Spectrograph Spectroscopy. Astrophys. J. 2002, 579, 774-799. [CrossRef]

53. Hillier, D.J.; Lanz, T.; Heap, S.R.; Hubeny, I.; Smith, L.J.; Evans, C.J.; Lennon, D.J.; Bouret, J.C. A Tale of Two Stars: The Extreme O7 Iaf+ Supergiant AV 83 and the OC7.5 III((f)) star AV 69. Astrophys. J. 2003, 588, $1039-1063$. [CrossRef]

54. Fullerton, A.W.; Massa, D.L.; Prinja, R.K. The Discordance of Mass-Loss Estimates for Galactic O-Type Stars. Astrophys. J. 2006, 637, 1025-1039. [CrossRef]

55. Neugent, K.F.; Massey, P.; Skiff, B.; Drout, M.R.; Meynet, G.; Olsen, K.A. Yellow Supergiants in the Small Magellanic Cloud: Putting Current Evolutionary Theory to the Test. Astrophys. J. 2010, 719, 1784-1795. [CrossRef]

56. Neugent, K.F.; Massey, P.; Skiff, B.; Meynet, G. Yellow and Red Supergiants in the Large Magellanic Cloud. Astrophys. J. 2012, 749, 177. [CrossRef] 
57. Humphreys, R.M.; Davidson, K.; Grammer, S.; Kneeland, N.; Martin, J.C.; Weis, K.; Burggraf, B. Luminous and Variable Stars in M31 and M33. I. The Warm Hypergiants and Post-red Supergiant Evolution. Astrophys. J. 2013, 773, 46. [CrossRef]

58. Humphreys, R.M.; Weis, K.; Davidson, K.; Bomans, D.J.; Burggraf, B. Luminous and Variable Stars in M31 and M33. II. Luminous Blue Variables, Candidate LBVs, Fe II Emission Line St ars, and Other Supergiants. Astrophys. J. 2014, 790, 48. [CrossRef]

59. Massey, P.; Neugent, K.F.; Smart, B.M. A Spectroscopic Survey of Massive Stars in M31 and M33. Astron. J. 2016, 152, 62. [CrossRef]

60. Humphreys, R.M.; Gordon, M.S.; Martin, J.C.; Weis, K.; Hahn, D. Luminous and Variable Stars in M31 and M33. IV. Luminous Blue Variables, Candidate LBVs, B[e] Supergiants, and the Warm Hypergiants: How to Tell Them Apart. Astrophys. J. 2017, 836, 64. [CrossRef]

61. Humphreys, R.M.; Davidson, K.; Hahn, D.; Martin, J.C.; Weis, K. Luminous and Variable Stars in M31 and M33. V. The Upper HR Diagram. Astrophys. J. 2017, 844, 40. [CrossRef]

62. Massey, P.; Silva, D.R.; Levesque, E.M.; Plez, B.; Olsen, K.A.; Clayton, G.C.; Meynet, G.; Maeder, A. Red Supergiants in the Andromeda Galaxy (M31). Astrophys. J. 2009, 703, 420-440. X/703/1/420. [CrossRef]

63. Drout, M.R.; Massey, P.; Meynet, G.; Tokarz, S.; Caldwell, N. Yellow Supergiants in the Andromeda Galaxy (M31). Astrophys. J. 2009, 703, 441-460. /703/1/441. [CrossRef]

64. Drout, M.R.; Massey, P.; Meynet, G. The Yellow and Red Supergiants of M33. Astrophys. J. 2012, 750, 97. [CrossRef]

65. Gordon, M.S.; Humphreys, R.M.; Jones, T.J. Luminous and Variable Stars in M31 and M33. III. The Yellow and Red Supergiants and Post-red Supergiant Evolution. Astrophys. J. 2016, 825, 50. [CrossRef]

66. Massey, P.; Evans, K.A. The Red Supergiant Content of M31. Astrophys. J. 2016, 826, 224. [CrossRef]

67. Massey, P.; Armandroff, T.E.; Pyke, R.; Patel, K.; Wilson, C.D. Hot, Luminous Stars in Selected Regions of NGC 6822, M31, and M33. Astron. J. 1995, 110, 2715-2745. [CrossRef]

68. Massey, P. Evolved Massive Stars in the Local Group. I. Identification of Red Supergiants in NGC 6822, M31, and M33. Astrophys. J. 1998, 501, 153-174. [CrossRef]

69. Bresolin, F.; Urbaneja, M.A.; Gieren, W.; Pietrzyński, G.; Kudritzki, R.P. VLT Spectroscopy of Blue Supergiants in IC 1613. Astrophys. J. 2007, 671, 2028-2039. [CrossRef]

70. Humphreys, R.M.; Aaronson, M.; Liebofsky, M.; McAlary, C.W.; Strom, S.E.; Capps, R.W. The luminosities of M supergiants and the distances to M 101, NGC 2403, and M 81. Astron. J. 1986, 91, 808-821. [CrossRef]

71. Humphreys, R.M.; Aaronson, M. The Visually Brightest Early-Type Supergiants in the Spiral Galaxies NGC 2403, M81, and M101. Astron. J. 1987, 94, 1156-1169. [CrossRef]

72. Zickgraf, F.-J.; Humphreys, R.M. A Stellar Content Survey of NGC 2403 and M81. Astron. J. 1991, 102, 113-133. [CrossRef]

73. Kudritzki, R.-P.; Urbaneja, M.A.; Bresolin, F.; Przybilla, N.; Gieren, W.; Pietrzyński, G. Quantitative Spectroscopy of 24 A Supergiants in the Sculptor Galaxy NGC 300: Flux-weighted Gravity-Luminosity Relationship, Metallicity, and Metallicity Gradient. Astrophys. J. 2008, 681, 269-289. [CrossRef]

74. Urbaneja, M.A. The Araucaria Project: The Local Group Galaxy WLM-Distance and Metallicity from Quantitative Spectroscopy of Blue Supergiants. Astrophys. J. 2008, 684, 118-135. [CrossRef]

75. Vivian, U.; Urbaneja, M.A.; Kudritzki, R.P.; Jacobs, B.A.; Bresolin, F.; Przybilla, N. A New Distance to M33 Using Blue Supergiants and the FGLR Method. Astrophys. J. 2009, 704, 1120-1134. [CrossRef]

76. Humphreys, R.M.; Davidson, K. The Most Luminous Stars. Science 1984, 223, 243-249. [CrossRef] [PubMed]

77. Instabilities in Luminous Early Type Stars; Henny, L., de Loore, C., Eds.; D. Reidel Publishing Company: Dordrecht, The Netherlands, 1987; Volume 136.

78. Physics of Luminous Blue Variables; Davidson, K., Moffat, A.F.J., Lamers, H.J.G.L.M., Eds.; Kluwer Academic Publishers: Dordrecht, The Netherlands; Boston, MA, USA, 1989, Volume 157.

79. Humphreys, R.M.; Weis, K.; Davidson, K.; Gordon, M.S. On the Social Traits of Luminous Blue Variables. Astrophys. J. 2016, 825, 64. [CrossRef]

80. Lamers, H.J.G.L.M.; Fitzpatrick, E.L. The Relationship between the Eddington Limit, the Observed Upper Luminosity Limit for Massive Stars, and the Luminous Blue Variables. Astrophys. J. 1988, 324, 279-287. [CrossRef] 
81. Glatzel, W.; Kiriakidis, M. Stability of Massive Stars and the Humphreys/Davidson Limit. MNRAS 1993, 263, 375-384. [CrossRef]

82. Ulmer, A.; Fitzpatrick, E.L. Revisiting the Modified Eddington Limit for Massive Stars. Astrophys. J. 1998, 504, 200-206. [CrossRef]

83. Humphreys, R.M.; Davidson, K. The Luminous Blue Variables: Astrophysical Geysers. PASP 1994, 106, 1025-1051. [CrossRef]

84. Smartt, S.J.; Eldridge, J.J.; Crockett, R.M.; Maund, J.R. The death of massive stars-I. Observational constraints on the progenitors of Type II-P supernovae. Mon. Not. R. Astron. Soc. 2009, 395, 1409-1437. [CrossRef]

85. Smartt, S.J. Observational Constraints on the Progenitors of Core-Collapse Supernovae: The Case for Missing High-Mass Stars. PASA 2015, 32. [CrossRef]

86. Jennings, Z.G.; Williams, B.F.; Murphy, J.W.; Dalcanton, J.J.; Gilbert, K.M.; Dolphin, A.E.; Weisz, D.R.; Fouesneau, M. The Supernova Progenitor Mass Distributions of M31 and M33: Further Evidence for an Upper Mass Limit. Astrophys. J. 2014, 795, 170. [CrossRef]

87. Ekstrom, S.; Georgy, C.; Eggenberger, P.; Meynet, G.; Mowlavi, N.; Wyttenbach, A.; Granada, A.; Decressin, T.; Hirschi, R.; Frischknecht, U.; et al. Grids of stellar models with rotation. I. Models from 0.8 to $120 \mathrm{M}_{\odot}$ at solar metallicity $(\mathrm{Z}=0.014)$. Astron. Astrophys. 2012, 537, A146. [CrossRef]

88. Bond, H.E. Hubble Space Telescope Imaging of the Outburst Site of M31 RV. II. No Blue Remnant in Quiescence. Astrophys. J. 2011, 737, 17. [CrossRef]

89. Davidson, K. Giant eruptions of very massive stars. J. Phys. Conf. Ser. 2016, 728. [CrossRef]

90. Riess, A.G.; Fliri, J.; Valls-Gabaud, D. Cepheid Period-Luminosity Relations in the Near-infrared and the Distance to M31 from the Hubble Space Telescope Wide Field Camera 3. Astrophys. J. 2012, 745, 156. . [CrossRef]

91. Scowcroft, V.; Bersier, D.; Mould, J.R.; Wood, P.R. The effect of metallicity on Cepheid magnitudes and the distance to M33. Mon. Not. Royal Astron. Soc. 2009, 396 1287-1296. [CrossRef]

92. Feast, M.W.; Whitelock, P.A.; Menzies, J.W.; Matsunaga, N. The Cepheid distance to the Local Group galaxy NGC 6822. Mon. Not. Royal Astron. Soc. 2012, 421, 2998-3003. [CrossRef]

93. Scowcroft, V.; Freedman, W.L.; Madore, B.F.; Monson, A.J.; Persson, S.E.; Seibert, M.; Rigby, J.R.; Melbourne, J. The Carnegie Hubble Program: The Infrared Leavitt Law in IC 1613. Astrophys. J. 2013, 773, 106. [CrossRef]

94. Freedman, W.L.; Madore, B.F.: Gibson, B.K.; Ferrarese, L.; Kelson, D.D.; Sakai, S.; Mould, J.R.; Kennicutt, R.C., Jr.; Ford, H.C.; Graham, J.A.; et al. Final Results from the Hubble Space Telescope Key Project to Measure the Hubble Constant. Astrophys. J. 2001, 553, 47-72. [CrossRef]

(C) 2019 by the author. Licensee MDPI, Basel, Switzerland. This article is an open access article distributed under the terms and conditions of the Creative Commons Attribution (CC BY) license (http:/ / creativecommons.org/licenses/by/4.0/). 